\title{
Exploring the Dynamic links between ICE BofA Yield Curves and First Bitcoin Capital Corp. Volatility using DECO-GARCH
}

Konstantinos Tsiaras

University of Ioannina, PhD University Campus, 54110, Ioannina, Greece

DOI: $10.36348 /$ sjef.2020.v04i12.001

| Received: 20.11.2020 | Accepted: 03.12.2020 | Published: 05.12.2020

*Corresponding author: Konstantinos Tsiaras

\section{Abstract}

This paper examines the time-varying conditional correlations between FIRST BITCOIN CAP and ICE BofA Sterling Zero Coupon markets. We apply ten bivariate DECO-GARCH models in order to capture potential contagion effects between the markets for the period 2007-2020. Empirical results reveal contagion during the under investigation period regarding the ten bivariate models, showing potential volatility transmission channels among the markets. Findings have crucial implications for policymakers who provide regulations for the above derivative markets and for investors, who invest long-term into FIRST BITCOIN CAP.

Key words: DECO-GARCH, Bitcoin market, financial contagion, equicorrelations, zero coupon market.

Copyright () 2020 The Author(s): This is an open-access article distributed under the terms of the Creative Commons Attribution 4.0 International License (CC BY-NC 4.0) which permits unrestricted use, distribution, and reproduction in any medium for non-commercial use provided the original author and source are credited.

\section{INTRODUCTION}

\section{We present the introduction as follows}

The main objective of this paper is to investigate how the FIRST BITCOIN CAP volatility shapes the distribution of ICE BofA Sterling Zero Coupon market returns. We contact this empirical study by using a multivariate DECO-GARCH model, which allows for the investigation of market interconnectedness, volatility transmission, and the dynamic correlation between market [1], as well as potential spillovers and contagion effects between markets.

This empirical study adds to the related literature by addressing the following questions, which are under-researched in the literature. Do Dynamic Conditional Correlations (DCCs) among those seemingly unrelated FIRST BITCOIN CAP and ten ICE BofA Sterling Zero Coupon markets exist? Are those DCCs volatile? How do those DCCs evolve over time? Are there contagion effects? Based on DCCs, do we observe interdependence?

The remainder of the paper is organized at follows: Section two reviews the literature. Section three describes the model and the data. Section four analyzes the empirical results, while Section gives concluding comments.

\section{LITERATURE REVIEW}

Since its introduction in 2009, Bitcoin is considered an alternative to mainstream currencies and has been called digital gold [2-6]. There is studies focus on Bitcoin volatility dynamics [7-12]. Other empirical studies investigate the spillover effects between Bitcoin and traditional financial assets [13-20]. Moreover, there are studies explore the interactions of Bitcoin derivative markets with other financial markets [21-25].

This paper addresses this literature gap by assessing to what extent FIRST BITCOIN CAP can have volatility spillover movements in the prices of ICE BofA Sterling Zero Coupon markets.

\section{METHODOLOGY}

Model description

First, we define conditional mean equation for the basic GARCH model:

$\mathrm{y}_{\mathrm{t}}=\mu+\varepsilon_{\mathrm{t}}$, with $\mathrm{t}=1, \ldots, \mathrm{T}$

where $\mu$ is constant and $\varepsilon_{\mathrm{t}}$ is standardized residuals described as:

$\varepsilon_{t}=\sqrt{h_{t}} u_{t}$, where $\varepsilon_{t} \sim N\left(0, H_{t}\right)$ and $u_{t}$ are i. i.d.

where $u_{t}$ is standardized errors and $h_{t}$ is conditional variance depending on $h_{t}$ and $\varepsilon_{t}$ for each market lagged one period, generated by the univariate $\operatorname{GARCH}(1,1)$ model [26]:

$h_{t}=\omega+a \varepsilon_{t-1}^{2}+b h_{t-1}$ 
where $\omega$ is constant, $a$ and $b$ are $\mathrm{ARCH}$ and $\mathrm{GARCH}$ effects.

The DECO model of Engle and Kelly [27] is defined as follows:

$\mathrm{H}_{\mathrm{t}}=\mathrm{D}_{\mathrm{t}} \mathrm{R}_{\mathrm{t}} \mathrm{D}_{\mathrm{t}}$

$R_{t}=\left(1-\rho_{t}\right) I_{N}+\rho_{t} J_{N x N}$

$\rho_{\mathrm{t}}=\frac{1}{\mathrm{~N}(\mathrm{~N}-1)} \sum_{\mathrm{i} \neq \mathrm{j}} \frac{\mathrm{q}_{\mathrm{ij}, \mathrm{t}}}{\sqrt{\mathrm{q}_{\mathrm{i} i, \mathrm{t}} \mathrm{q}_{\mathrm{jj}, \mathrm{t}}}}$

And $\rho_{t}$ is the equicorrelation, with $q_{i j, t}$ being the $i, j$ th element of $Q_{t}$ given by:

$\mathrm{Q}_{\mathrm{t}}=(1-\alpha-\beta) \overline{\mathrm{Q}}+\alpha \mathrm{u}_{\mathrm{t}-1} \mathrm{u}_{\mathrm{t}-1}^{\prime}+\beta \mathrm{Q}_{\mathrm{t}-1}$,

$\mathrm{u}_{\mathrm{it}}=\varepsilon_{\mathrm{it}} \sqrt{\mathrm{h}_{\mathrm{iit}}}, \mathrm{Q}$ is the $\mathrm{N} \times \mathrm{N}$ unconditional variance matrix of $u_{t}$, and $\alpha$ and $\beta$ are nonnegative scalar parameters, satisfying $\alpha+\beta<1$. $\mathrm{I}_{\mathrm{N}}$ denotes the $\mathrm{N}$ dimensional identity matrix and $\mathrm{J}_{\mathrm{NxN}}$ is an $\mathrm{N} \mathrm{x} \mathrm{N}$ matrix of ones. Engle and Kelly [27] state that

$\mathrm{R}_{\mathrm{t}}^{-1}$ exists if $\rho_{\mathrm{t}} \neq 1$ and $\rho_{\mathrm{t}} \neq-1 /(\mathrm{N}-1)$ and

$\mathrm{R}_{\mathrm{t}}$ is a positive definite if $-\frac{1}{\mathrm{~N}-1}<\rho_{\mathrm{t}}>1$.

\section{Data description}

We use daily data for FIRST BITCOIN CAP., ICE BofA 5 Year Sterling Tradeable Zero Coupon Swap Index - Tota, ICE BofA 10 Year Sterling Tradeable Zero Coupon Swap Index - Tot, ICE BofA 15 Year Sterling Tradeable Zero Coupon Swap Index Tot, ICE BofA 20 Year Sterling Tradeable Zero
Coupon Swap Index - Tot, ICE BofA 25 Year Sterling Tradeable Zero Coupon Swap Index - Tot, ICE BofA 30 Year Sterling Tradeable Zero Coupon Swap Index Tot, ICE BofA 35 Year Sterling Synthetic Zero Coupon Swap Index - Pri, ICE BofA 35 Year Sterling Tradeable Zero Coupon Swap Index - Tot, ICE BofA 40 Year Sterling Synthetic Zero Coupon Swap Index Pri and ICE BofA 45 Year Sterling Synthetic Zero Coupon Swap Index - Pri. The study period runs from $20^{\text {th }}$ December 2007 through $28^{\text {th }}$ August 2020. All prices have been extracted from Datastream ${ }^{\circledR}$ Database. For each market we use 2212 observations. We calculate the continuously compounded daily logarithmic returns by taking the difference in the logarithms of two consecutive prices.

Figure 1 displays the daily dynamic behaviour of the markets between $20^{\text {th }}$ December 2007 and $28^{\text {th }}$ August 2020. From this figure, we can see that all of the series exhibit an important variability. Interestingly, we observe that all markets are following a common upward trend during the period.

Figure 2 illustrates the return behaviour of the markets. Returns dynamics present sudden ups and downs indicating the existence of heteroskedasticity. To analyse the effects of sudden changes in returns and volatility, we use the DECO-GARCH process.

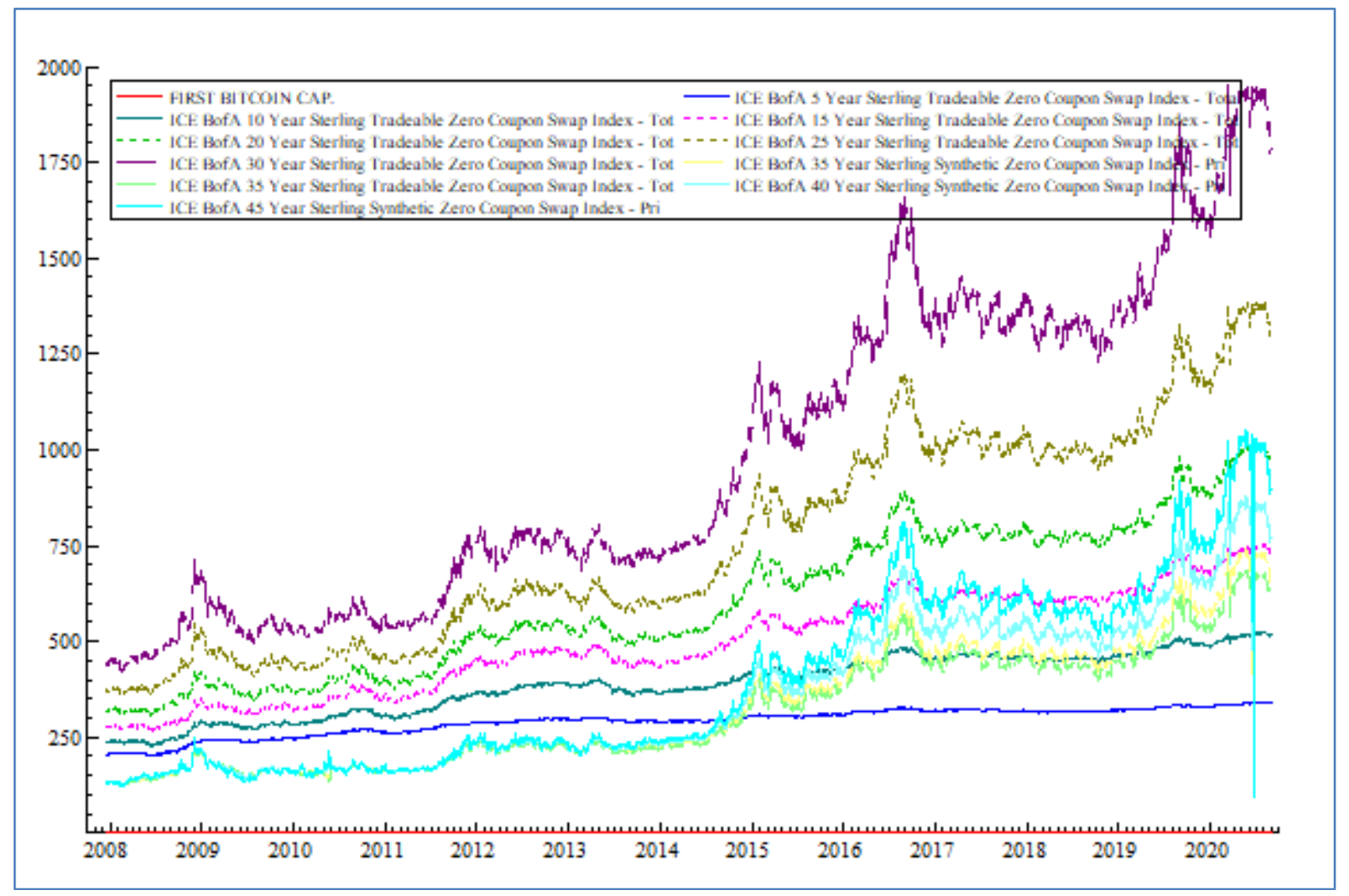

Fig-1: Actual series of the markets 


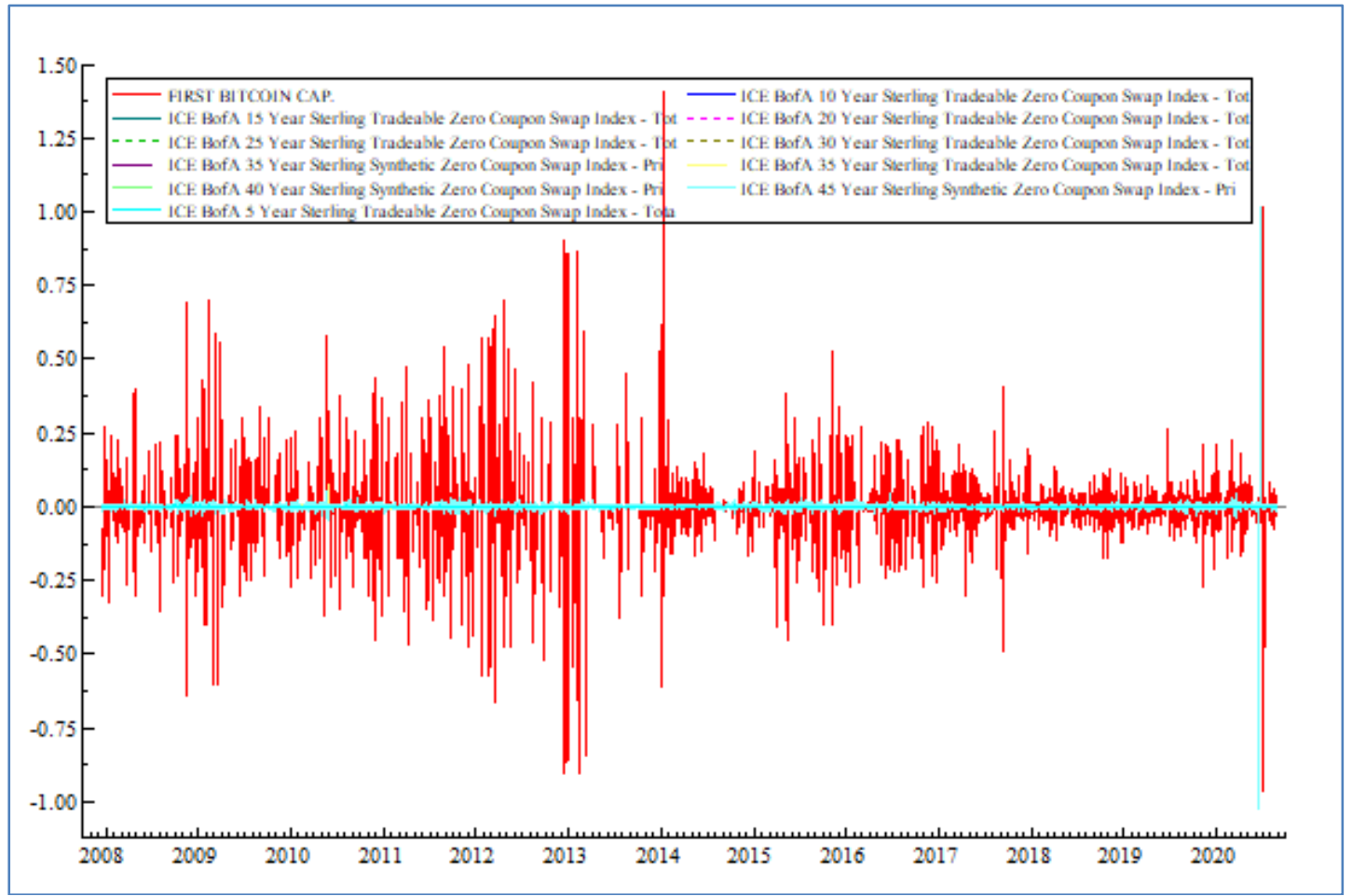

Fig-2: Actual series of the logarithmic returns of the markets

\section{RESULTS AND DISCUSSION}

This section discusses the results of the estimation for the spillovers among the markets. It then analyses the dynamic equicorrelation results in order to extract important drawbacks.

\section{Results of the DECO-GARCH $(1,1)$ model}

Table 1 to 4 present the estimated of the GARCH $(1,1)$ process. The $\mu$ is significant for ICE BofA 5 Year Sterling Tradeable Zero Coupon Swap Index - Tota, ICE BofA 10 Year Sterling Tradeable
Zero Coupon Swap Index - Tot, ICE BofA 15 Year Sterling Tradeable Zero Coupon Swap Index - Tot, ICE BofA 20 Year Sterling Tradeable Zero Coupon Swap Index - Tot and ICE BofA 25 Year Sterling Tradeable Zero Coupon Swap Index - Tot. Looking at the conditional variance equation estimates, constant $(\omega)$ is significant for all markets except the case of ICE BofA 5 Year Sterling Tradeable Zero Coupon Swap Index Tota. It is worth noting that the significance of the $\mathrm{ARCH}$ (a) and GARCH (b) terms indicates the appropriateness of the DECO-GARCH model.

Table-1: Estimates of univariate GARCH $(1,1)$ model

\begin{tabular}{|l|l|l|l|}
\hline & $\begin{array}{l}\text { FIRST BITCOIN } \\
\text { CAP. }\end{array}$ & $\begin{array}{l}\text { ICE BofA 5 Year } \\
\text { Sterling Tradeable } \\
\text { Zero Coupon Swap } \\
\text { Index - Tota }\end{array}$ & $\begin{array}{l}\text { ICE BofA 10 Year } \\
\text { Sterling Tradeable } \\
\text { Zero Coupon Swap } \\
\text { Index - Tot }\end{array}$ \\
\hline constant $(\mu)$ & $-0,001484$ & $0,0001534^{* * *}$ & $0,0001857^{* *}$ \\
\hline t-Statistic & $-0,3360$ & 3,671 & 2,449 \\
\hline p-Value & 0,7370 & 0,0003 & 0,0145 \\
\hline constant $(\omega)$ & $4,636595^{*}$ & 0,014227 & $0,046613^{*}$ \\
\hline t-Statistic & 1,472 & 0,7564 & 1,129 \\
\hline p-Value & 0,1415 & 0,4497 & 0,2593 \\
\hline ARCH $(a)$ & $0,044181^{*}$ & $0,042732^{*}$ & $0,047095^{* *}$ \\
\hline t-Statistic & 1,876 & 1,567 & 2,341 \\
\hline p-Value & 0,0610 & 0,1177 & 0,0195 \\
\hline GARCH $(b)$ & $0,921671^{* * *}$ & $0,948218^{* * *}$ & $0,944548^{* * *}$ \\
\hline t-Statistic & 24,30 & 26,05 & 39,63 \\
\hline p-Value & 0,0000 & 0,0000 & 0,0000 \\
\hline
\end{tabular}


Table-2: Estimates of univariate GARCH $(1,1)$ model

\begin{tabular}{|l|l|l|l|}
\hline & $\begin{array}{l}\text { ICE BofA 15 Year } \\
\text { Sterling Tradeable } \\
\text { Zero Coupon } \\
\text { Swap Index - Tot }\end{array}$ & $\begin{array}{l}\text { ICE Bof A 20 Year } \\
\text { Sterling Tradeable } \\
\text { Zero Coupon Swap } \\
\text { Index - Tot }\end{array}$ & $\begin{array}{l}\text { ICE BofA 25 Year } \\
\text { Sterling Tradeable } \\
\text { Zero Coupon Swap } \\
\text { Index - Tot }\end{array}$ \\
\hline constant $(\mu)$ & $0,000197 *$ & $0,000184^{*}$ & $0,000153^{*}$ \\
\hline t-Statistic & 1,891 & 1,468 & 1,057 \\
\hline p-Value & 0,0590 & 0,1426 & 0,2909 \\
\hline constant $(\omega)$ & $0,142220^{*}$ & $0,300802^{*}$ & $0,477410^{*}$ \\
\hline t-Statistic & 1,229 & 1,189 & 1,089 \\
\hline p-Value & 0,2193 & 0,2348 & 0,2764 \\
\hline ARCH $(a)$ & $0,064121^{* *}$ & $0,082059 * *$ & $0,085272^{* *}$ \\
\hline t-Statistic & 2,331 & 2,163 & 2,064 \\
\hline p-Value & 0,0200 & 0,0309 & 0,0394 \\
\hline GARCH $(b)$ & $0,923872 * * *$ & $0,901662^{* * *}$ & $0,894470^{* * *}$ \\
\hline t-Statistic & 28,79 & 19,52 & 15,80 \\
\hline p-Value & 0,0000 & 0,0000 & 0,0000 \\
\hline
\end{tabular}

Table-3: Estimates of univariate $\operatorname{GARCH}(1,1)$ model

\begin{tabular}{|l|l|l|l|}
\hline & $\begin{array}{l}\text { ICE Bof A 30 Year } \\
\text { Sterling Tradeable } \\
\text { Zero Coupon } \\
\text { Swap Index - Tot }\end{array}$ & $\begin{array}{l}\text { ICE Bof A 35 Year } \\
\text { Sterling Synthetic } \\
\text { Zero Coupon Swap } \\
\text { Index - Pri }\end{array}$ & $\begin{array}{l}\text { ICE BofA 35 Year } \\
\text { Sterling Tradeable } \\
\text { Zero Coupon Swap } \\
\text { Index - Tot }\end{array}$ \\
\hline constant $(\mu)$ & 0,000133 & 0,000107 & 0,000104 \\
\hline t-Statistic & 0,7809 & 0,5335 & 0,5185 \\
\hline p-Value & 0,4351 & 0,5938 & 0,6043 \\
\hline constant $(\omega)$ & $0,712933^{*}$ & $1,593138^{*}$ & $1,583579^{*}$ \\
\hline t-Statistic & 1,005 & 1,438 & 1,448 \\
\hline p-Value & 0,3153 & 0,1508 & 0,1481 \\
\hline ARCH $(a)$ & $0,087256^{*}$ & $0,146342^{* * *}$ & $0,145710^{* * *}$ \\
\hline t-Statistic & 1,908 & 3,828 & 3,821 \\
\hline p-Value & 0,0568 & 0,0001 & 0,0001 \\
\hline GARCH $(b)$ & $0,889503^{* * *}$ & $0,834125^{* * *}$ & $0,834892^{* * *}$ \\
\hline t-Statistic & 13,42 & 25,35 & 25,69 \\
\hline p-Value & 0,0000 & 0,0000 & 0,0000 \\
\hline
\end{tabular}

Table-4: Estimates of univariate GARCH $(1,1)$ model

\begin{tabular}{|l|l|l|}
\hline & $\begin{array}{l}\text { ICE BofA 40 Year Sterling } \\
\text { Synthetic Zero Coupon } \\
\text { Swap Index - Pri }\end{array}$ & $\begin{array}{l}\text { ICE BofA 45 Year Sterling } \\
\text { Synthetic Zero Coupon } \\
\text { Swap Index - Pri }\end{array}$ \\
\hline constant $(\mu)$ & 0,000134 & 0,000118 \\
\hline t-Statistic & 0,5622 & 0,4240 \\
\hline p-Value & 0,5742 & 0,6717 \\
\hline constant $(\omega)$ & $2,860192^{*}$ & $4,729412^{* *}$ \\
\hline t-Statistic & 1,191 & 2,184 \\
\hline p-Value & 0,2341 & 0,0293 \\
\hline ARCH $(a)$ & $0,092205^{*} *$ & $0,119953^{* * *}$ \\
\hline t-Statistic & 2,177 & 3,908 \\
\hline p-Value & 0,0298 & 0,0001 \\
\hline GARCH $(b)$ & $0,853310^{* * *}$ & $0,822254 * * *$ \\
\hline t-Statistic & 10,26 & 18,38 \\
\hline p-Value & 0,0000 & 0,0000 \\
\hline
\end{tabular}


Figure 3 plots our conditional variance estimations. Findings present considerable time variation. More importantly, variances do not show a tendency to increase over time. In addition, we observe periods of relatively low volatility. The time pattern of major economic crises in figures 1 and 2 is consistent with the results in figure 3 .

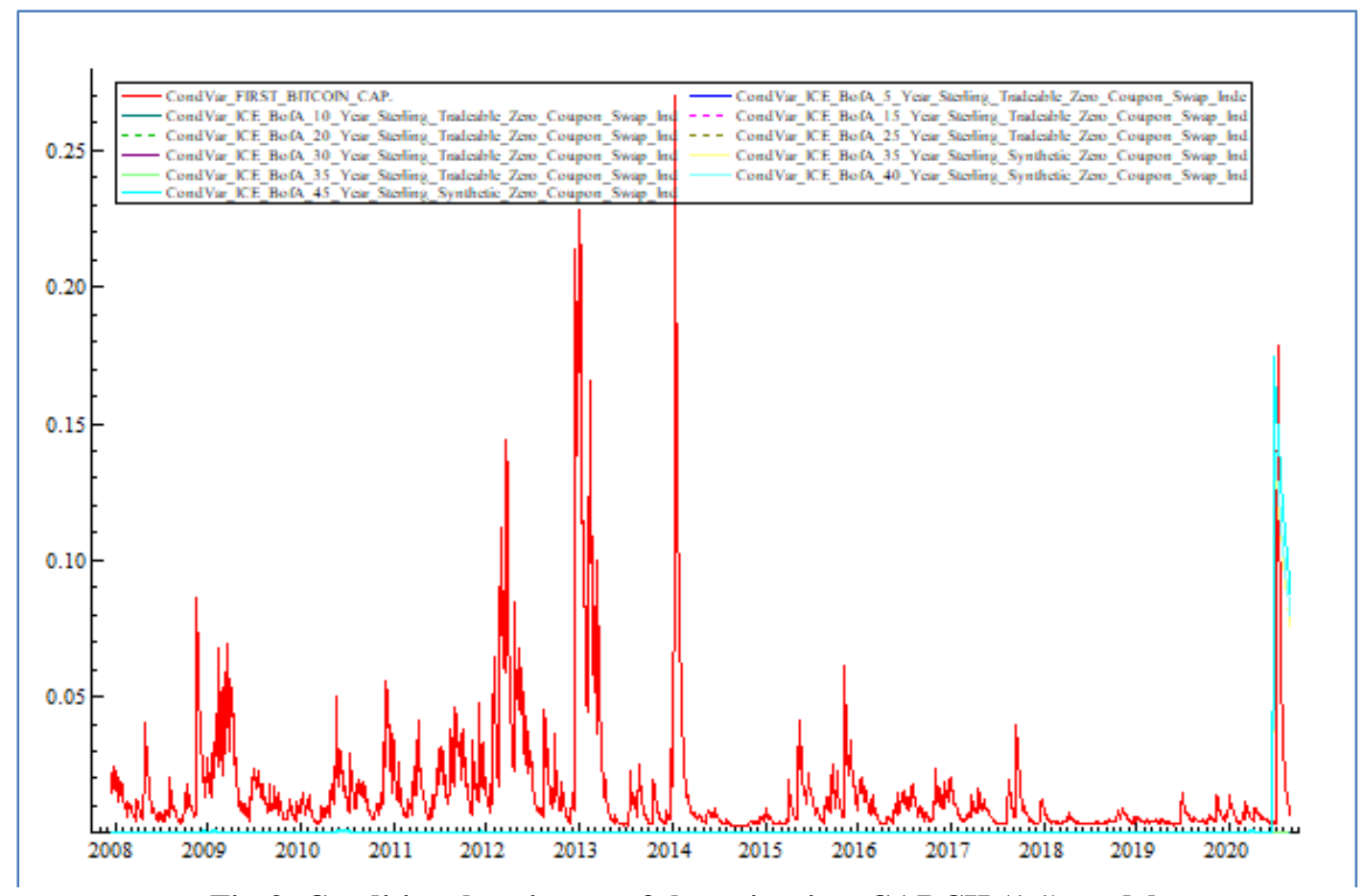

Fig-3: Conditional variances of the univariate GARCH $(1,1)$ model

The estimates of the bivariate DECO model summarized in tables 5 to 8 show significant average correlation except the cases of FIRST BITCOIN CAP.ICE BofA 5 Year Sterling Tradeable Zero Coupon Swap Index - Tota, FIRST BITCOIN CAP.- ICE BofA 10 Year Sterling Tradeable Zero Coupon Swap Index - Tot, FIRST BITCOIN CAP.- ICE BofA 15 Year Sterling Tradeable Zero Coupon Swap Index - Tot, FIRST BITCOIN CAP.- ICE BofA 20 Year Sterling Tradeable
Zero Coupon Swap Index - Tot and FIRST BITCOIN CAP.- ICE BofA 25 Year Sterling Tradeable Zero Coupon Swap Index - Tot. The DECO-GARCH estimates reveal significance of the parameters $\alpha$ and $\beta$, suggesting strong ARCH and GARCH effects and that the markets are potentially integrated. Furthermore, the estimates of the degrees of freedom (v) and of the loglikelihood are stated.

Table-5: Estimates of the bivariate DECO-GARCH $(1,1)$ model, degrees of freedom, log-likelihood

\begin{tabular}{|l|l|l|l|}
\hline & $\begin{array}{l}\text { FIRST BITCOIN CAP.- } \\
\text { ICE BofA 5 Year Sterling } \\
\text { Tradeable Zero Coupon } \\
\text { Swap Index - Tota }\end{array}$ & $\begin{array}{l}\text { FIRST BITCOIN CAP.- ICE } \\
\text { Bof 10 Year Sterling } \\
\text { Tradeable Zero Coupon } \\
\text { Swap Index - Tot }\end{array}$ & $\begin{array}{l}\text { FIRST BITCOIN CAP.- } \\
\text { ICE BofA 15 Year Sterling } \\
\text { Tradeable Zero Coupon } \\
\text { Swap Index - Tot }\end{array}$ \\
\hline rho & $-0,002859$ & 0,001838 & 0,008989 \\
\hline t-Statistic & $-0,08735$ & 0,05416 & 0,2711 \\
\hline p-Value & 0,9304 & 0,9568 & 0,7864 \\
\hline alpha $(\alpha)$ & $0,026574^{*}$ & $0,025709 * *$ & $0,023206^{*}$ \\
\hline t-Statistic & 1,685 & 2,009 & 1,766 \\
\hline p-Value & 0,0925 & 0,0449 & 0,0778 \\
\hline beta $(\beta)$ & $0,814586^{* * *}$ & $0,850261^{* * *}$ & $0,852403^{* * *}$ \\
\hline t-Statistic & 11,65 & 17,91 & 15,55 \\
\hline p-Value & 0,0000 & 0,0000 & 0,0000 \\
\hline degrees of freedom $(\mathrm{df})$ & $3,170076^{* * *}$ & $3,328928 * * *$ & $3,372309^{* * *}$ \\
\hline t-Statistic & 17,81 & 16,80 & 16,68 \\
\hline p-Value & 0,0000 & 0,0000 & 0,0000 \\
\hline log-likelihood & 4573,419 & 4128,958 & 3877,815 \\
\hline
\end{tabular}


Table-6: Estimates of the bivariate DECON-GARCH $(1,1)$ model, degrees of freedom, log-likelihood

\begin{tabular}{|l|l|l|l|}
\hline & $\begin{array}{l}\text { FIRST BITCOIN CAP.- } \\
\text { ICE BofA 20 Year Sterling } \\
\text { Tradeable Zero Coupon } \\
\text { Swap Index - Tot }\end{array}$ & $\begin{array}{l}\text { FIRST BITCOIN CAP.- } \\
\text { ICE BofA 25 Year Sterling } \\
\text { Tradeable Zero Coupon } \\
\text { Swap Index - Tot }\end{array}$ & $\begin{array}{l}\text { FIRST BITCOIN CAP.- } \\
\text { ICE BofA 30 Year Sterling } \\
\text { Tradeable Zero Coupon } \\
\text { Swap Index - Tot }\end{array}$ \\
\hline rho & 0,015834 & 0,026143 & $0,038563 *$ \\
\hline t-Statistic & 0,4955 & 0,8479 & 1,317 \\
\hline p-Value & 0,6204 & 0,3968 & 0,1882 \\
\hline alpha $(\alpha)$ & $0,019629 *$ & $0,016272^{*}$ & $0,010265^{*}$ \\
\hline t-Statistic & 1,626 & 1,552 & 1,143 \\
\hline p-Value & 0,1045 & 0,1211 & 0,2533 \\
\hline beta $(\beta)$ & $0,855940^{* * *}$ & $0,859460 * * *$ & $0,870001 * * *$ \\
\hline t-Statistic & 15,27 & 14,78 & 14,73 \\
\hline p-Value & 0,0000 & 0,0000 & 0,0000 \\
\hline degrees of freedom $(\mathrm{df})$ & $3,389212^{* * *}$ & $3,400766 * * *$ & $3,339197 * * *$ \\
\hline t-Statistic & 16,31 & 16,08 & 16,47 \\
\hline p-Value & 0,0000 & 0,0000 & 0,0000 \\
\hline log-likelihood & 3724,189 & 3616,965 & 3520,235 \\
\hline
\end{tabular}

Table-7: Estimates of the bivariate DECO-GARCH $(1,1)$ model, degrees of freedom, log-likelihood

\begin{tabular}{|l|l|l|}
\hline & $\begin{array}{l}\text { FIRST BITCOIN CAP.- } \\
\text { ICE BofA 35 Year Sterling } \\
\text { Synthetic Zero Coupon } \\
\text { Swap Index - Pri }\end{array}$ & $\begin{array}{l}\text { FIRST BITCOIN CAP.- } \\
\text { ICE BofA 35 Year Sterling } \\
\text { Tradeable Zero Coupon } \\
\text { Swap Index - Tot }\end{array}$ \\
\hline rho & $0,035566^{*}$ & $0,033827 *$ \\
\hline t-Statistic & 1,279 & 1,214 \\
\hline p-Value & 0,2014 & 0,2250 \\
\hline alpha $(\alpha)$ & $0,010390^{*}$ & $0,010857^{*}$ \\
\hline t-Statistic & 1,078 & 1,100 \\
\hline p-Value & 0,2812 & 0,2719 \\
\hline beta $(\beta)$ & $0,844724^{* * *}$ & $0,840172 * * *$ \\
\hline t-Statistic & 14,65 & 14,95 \\
\hline p-Value & 0,0000 & 0,0000 \\
\hline degrees of freedom $(\mathrm{df})$ & $3,114374 * * *$ & $3,117646^{* * *}$ \\
\hline t-Statistic & 19,56 & 19,56 \\
\hline p-Value & 0,0000 & 0,0000 \\
\hline log-likelihood & 3406,149 & 3404,890 \\
\hline
\end{tabular}

Table-8: Estimates of the bivariate DECO-GARCH $(1,1)$ model, degrees of freedom, log-likelihood

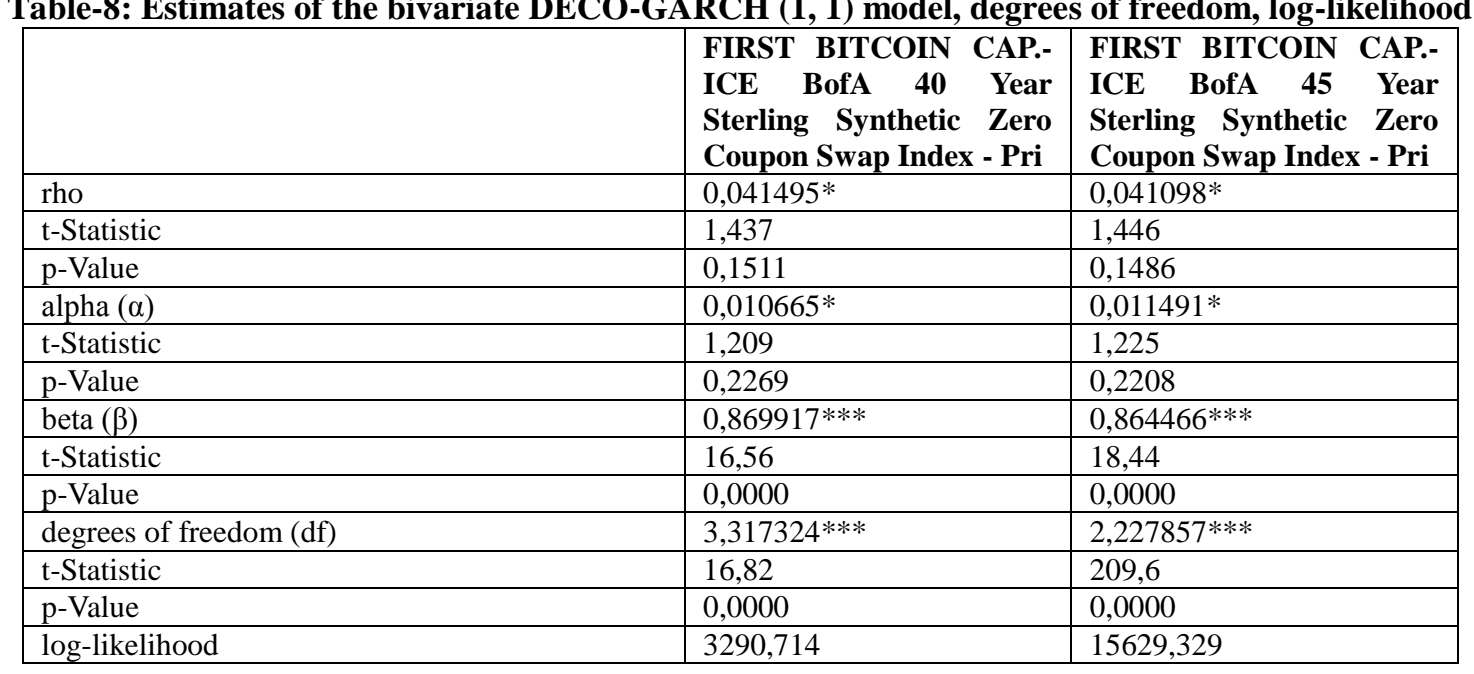

The diagnostic tests summarized in tables 9 to 12 show no evidence of misspecification in DECOGARCH model. In fact, $x^{2}(12)$ statistic results suggest the rejection of the null hypothesis of no spillovers at $1 \%$ significance level. Furthermore, Ljuing-Box test statistics [28, 29] for the standardized residuals and the squared standardized residuals show evidence of no serial autocorrelation. Moreover, the selected AIC and SIC information criteria are stated. 
Table-9: Diagnostic tests and information criteria

\begin{tabular}{|l|l|l|l|}
\hline & $\begin{array}{l}\text { FIRST BITCOIN CAP.- } \\
\text { ICE BofA 5 Year Sterling } \\
\text { Tradeable Zero Coupon } \\
\text { Swap Index - Tota }\end{array}$ & $\begin{array}{l}\text { FIRST BITCOIN CAP.- } \\
\text { ICE BofA 10 Year Sterling } \\
\text { Tradeable Zero Coupon } \\
\text { Swap Index - Tot }\end{array}$ & $\begin{array}{l}\text { FIRST BITCOIN CAP.- ICE } \\
\text { BofA 15 Year Sterling } \\
\text { Tradeable Zero Coupon Swap } \\
\text { Index - Tot }\end{array}$ \\
\hline $\mathrm{x}^{2}(4)$ & $583,31 * *$ & $576,14 * *$ & $580,91 * *$ \\
\hline p-Value & 0,0000 & 0,0000 & 0,0000 \\
\hline Hosking $^{2}(50)$ & 204,287 & 222,097 & 229,738 \\
\hline p-Value & 0,3646360 & 0,1154208 & 0,0605781 \\
\hline Li-McLeod $^{2}(50)$ & 204,617 & 221,776 & 229,120 \\
\hline p-Value & 0,3585681 & 0,1183559 & 0,0640347 \\
\hline Akaike & $-12,514180$ & $-11,294810$ & $-10,605803$ \\
\hline Schwarz & $-12,438597$ & $-11,219226$ & $-10,530220$ \\
\hline
\end{tabular}

Table -10: Diagnostic tests and information criteria

\begin{tabular}{|l|l|l|l|}
\hline & $\begin{array}{l}\text { FIRST BITCOIN CAP.- ICE } \\
\text { BofA 20 Year Sterling } \\
\text { Tradeable Zero Coupon Swap } \\
\text { Index - Tot }\end{array}$ & $\begin{array}{l}\text { FIRST BITCOIN CAP.- } \\
\text { ICE BofA 25 Year Sterling } \\
\text { Tradeable Zero Coupon } \\
\text { Swap Index - Tot }\end{array}$ & $\begin{array}{l}\text { FIRST BITCOIN CAP.- } \\
\text { ICE BofA 30 Year Sterling } \\
\text { Tradeable Zero Coupon } \\
\text { Swap Index - Tot }\end{array}$ \\
\hline $\mathrm{x}^{2}(4)$ & $582,90^{* *}$ & $580,86^{* *}$ & $586,87 * *$ \\
\hline p-Value & 0,0000 & 0,0000 & 0,0000 \\
\hline Hosking $^{2}(50)$ & 229,616 & 219,046 & 220,971 \\
\hline p-Value & 0,0612462 & 0,1455920 & 0,1259584 \\
\hline Li-McLeod $(50)$ & 229,171 & 219,141 & 221,185 \\
\hline p-Value & 0,0637395 & 0,1445738 & 0,1239035 \\
\hline Akaike & $-10,184333$ & $-9,890166$ & $-9,62788$ \\
\hline Schwarz & $-10,108750$ & $-9,814583$ & $-9,54205$ \\
\hline
\end{tabular}

Table-11: Diagnostic tests and information criteria

\begin{tabular}{|l|l|l|}
\hline & $\begin{array}{l}\text { FIRST BITCOIN CAP.- } \\
\text { ICE BofA 35 Year Sterling } \\
\text { Synthetic Zero Coupon } \\
\text { Swap Index - Pri }\end{array}$ & $\begin{array}{l}\text { FIRST BITCOIN CAP.- } \\
\text { ICE BofA 35 Year Sterling } \\
\text { Tradeable Zero Coupon } \\
\text { Swap Index - Tot }\end{array}$ \\
\hline $\mathrm{x}^{2}(4)$ & $809,62^{* *}$ & $809,57 * *$ \\
\hline p-Value & 0,0000 & 0,0000 \\
\hline Hosking ${ }^{2}(50)$ & 206,137 & 205,418 \\
\hline p-Value & 0,3311230 & 0,3439831 \\
\hline Li-McLeod $^{2}(50)$ & 207,026 & 206,326 \\
\hline p-Value & 0,3155224 & 0,3277830 \\
\hline Akaike & $-9,311795$ & $-9,308339$ \\
\hline Schwarz & $-9,236212$ & $-9,232756$ \\
\hline
\end{tabular}

Table-12: Diagnostic tests and information criteria

\begin{tabular}{|l|l|l|}
\hline & $\begin{array}{l}\text { FIRST BITCOIN CAP.- } \\
\text { ICE BofA 40 Year Sterling } \\
\text { Synthetic Zero Coupon } \\
\text { Swap Index - Pri }\end{array}$ & $\begin{array}{l}\text { FIRST BITCOIN CAP.- } \\
\text { ICE BofA 45 Year Sterling } \\
\text { Synthetic Zero Coupon } \\
\text { Swap Index - Pri }\end{array}$ \\
\hline $\mathrm{x}^{2}(4)$ & $590,68 * *$ & $591,92 * *$ \\
\hline p-Value & 0,0000 & 0,0000 \\
\hline Hosking $^{2}(50)$ & 227,455 & 227,598 \\
\hline p-Value & 0,0741387 & 0,0732200 \\
\hline Li-McLeod $^{2}(50)$ & 227,231 & 227,287 \\
\hline p-Value & 0,0755868 & 0,0752231 \\
\hline Akaike & $-8,995101$ & $-8,601517$ \\
\hline Schwarz & $-8,919517$ & $-8,525934$ \\
\hline
\end{tabular}

Dynamic equicorrelation estimates

Figure 4 displays the dynamic equicorrelation for the estimated bivariate DECO-GARCH model. As shown in this figure, we observe positive time-varying equicorrelations for all the pairs of markets over the period 2007-2020. This result reveals that investor should rebalance their portfolio choices. The presence of positive equicorrelations underscores the integration between the pairs of markets during the under investigation period. Graphically, we can identify the effects of major economic events by taking into consideration the picks and troughs i.e. (1) the Lehman 
Brother bankruptcy (15/9/2008), (2) the USA presidential election (4/11/2008), (3) the Standard \& Poor's credit rating agency downgrading of the credit rating for the USA from AAA to AA+ (5/8/2011), and the Covid-19 pandemic (03/2020).

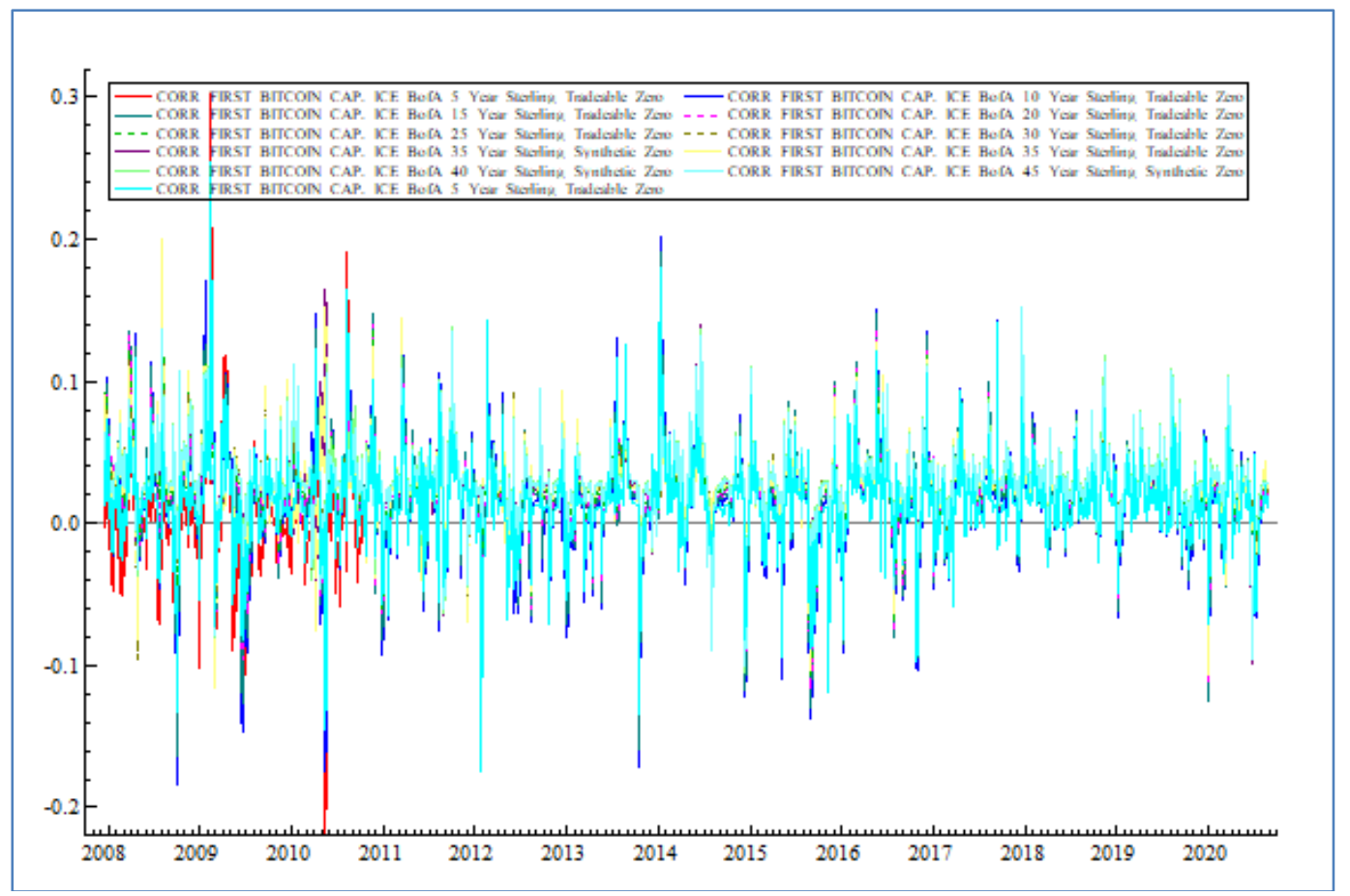

Fig-4: Dynamic equicorrelations of the bivariate DECO-GARCH $(1,1)$ model

\section{CONCLUSION}

The aim of this paper is to examine the volatility spillover effects between FIRST BITCOIN CAP and ten ICE BofA Sterling Zero Coupon markets. We used daily data for the period 2007 - 2020, which includes several major economic crises. We use the bivariate DECO-GARCH process. For comparison purposed, we estimate the equicorrelations. To the best of our knowledge, this is the first time in the literature; an empirical study has analyzed the volatility dynamics among the FIRST BITCOIN CAP and ICE BofA Sterling Zero Coupon markets using the DECOGARCH model.

Our results find significant spillover effects. More interestingly, the results show positive equicorrelation between all the pairs of markets during the period. The results have important implications for investors, portfolio managers and policy makers. Investors and portfolio managers should diversify their portfolios in bearish times when their investing is dealing with positive equicorrelation. Policy makers should re-examine the regulations of the under investigation derivate markets during future economic crises.

\section{REFERENCES}

1. Forbes, K. J., \& Rigobon, R. (2002). No Contagion, Only Interdependence: Measuring Stock Market Comovements. The Journal of Finance, 57(5), 2223-2261.
2. Akhtaruzzaman M., Senso, A., \& Corbet, S. (2019). The influence of Bitcoin on Portfolio Diversification and Design. Finance Research Letters.

3. Aalborg, H., Aarhus, P. M., \& de Vries J. E. (2019). What can explain the price, volatility and trading volume of bitcoin? Finance Research Letters, 29, 255-65.

4. Bouoiyour, J., \& Refk, S. (2015). What does bitcoin look like? Annals of Economics and Finance, 16(2), 449-92.

5. Corbet, S., Brian, L., Maurice, P., \& Samuel, V. (2018). Bitcoin futures-What use are they? Economics Letters, 172, 23-27.

6. Yermack, D. (2015). Is bitcoin a real currency? An economic appraisal. Handbook of Digital Currency, 31-43. DOI: 10.1016/B978-0-12-802117-0.000023.

7. Katsiampa, P., S. Corbet., \& Lucey, B. (2019). Volatility spillover effects in leading cryptocurrencies: A BEKK-MGARCH analysis. Finance Research Letters, 29, 68-74.

8. Bouri, E., Peter, M., Georges, A., David, R., \& Hagfors, L.I. (2017). On the hedge and safe haven properties of bitcoin: Is it really more than a diversifier? Finance Research Letters, 20, 192-98.

9. Charles, A., \& Olivier, D. (2019). Volatility estimation for bitcoin: Replication and robustness. International Economics, 157, 23-32. 
10. Dyhrberg, A. H. (2016). Bitcoin, gold and the dollar-A garch volatility analysis. Finance Research Letters, 16, 85-92.

11. Katsiampa, P. (2017). Volatility estimation for bitcoin: A comparison of garch models. Economics Letters, 158, 3-6.

12. Wu, S., Mu, T., Zhongyi, Y., \& Abdelkader, D. (2019). Does gold or bitcoin hedge economic policy uncertainty? Finance Research Letters, 31, 171-78.

13. Selmi, R., Mensi, W., Hammoudeh, S., \& Bouoiyour, J. (2018). Is Bitcoin a hedge, a safe haven or a diversifier for oil price movements? a comparison with gold. Energy Economics, 74, 787-801.

14. Dastgir, S., Ender, D., Gareth, D., Giray, G., \& Chi, K. M. L. (2019). The causal relationship between bitcoin attention and bitcoin returns: Evidence from the copula-based granger causality test. Finance Research Letters, 28, 160-64.

15. Kristoufek, L. (2013). Bitcoin meets google trends and wikipedia: Quantifying the relationship between phenomena of the internet era. Scientific Reports, 3.

16. Panagiotidis, T., Thanasis, S., \& Orestis, V. (2019). The effects of markets, uncertainty and search intensity on bitcoin returns. International Review of Financial Analysis, 63, 220-42.

17. Dyhrberg, A. H. (2016). Hedging capabilities of bitcoin. Is it the virtual gold? Finance Research Letters, 16, 139-44.

18. Bouri, E., Rangan G., Aviral, K.T., \& David, R. (2017). Does bitcoin hedge global uncertainty? Evidence from wavelet-based quantile-in-quantile regressions. Finance Research Letters, 23, 87-95.

19. Demir, E., Giray, G., Chi, K. M. L., \& Samuel, A. V. (2018). Does economic policy uncertainty predict the bitcoin returns? an empirical investigation. Finance Research Letters, 26, 14549.
20. Liu, R., Shanfeng, W., Zili, Z., \& Xuejun, Z. (2019). Is the introduction of futures responsible for the crash of bitcoin? Finance Research Letters, 34.

21. Akyildirim, E., Corbet, S., Katsiampa, P., Kellard, N., \& Sensoy, A. (2019). The development of bitcoin futures: Exploring the interactions between cryptocurrency derivatives. Finance Research Letters Available online at:

22. Kim, W. J. L., \& Kyungwon, K. (2019). The effects of the introduction of bitcoin futures on the volatility of bitcoin returns. Finance Research Letters, 33.

23. Sebastião, H., \& Pedro, G. (2019). Bitcoin futures: An effective tool for hedging cryptocurrencies. Finance Research Letters, 33.

24. Tsiaras, K. (2020). Dynamic relationship between future FOREX markets in the post Global Financial Crisis. Journal of Quantitative Methods, 4(1), 3052.

25. Tsiaras, K. (2020). Volatility spillover and contagion effects between eurodollar future and zero coupons markets: evidence from italy. The European Journal of Applied Economics, 17(2), 67-88.

26. Engle, R.F. (2002). Dynamic conditional correlation-a simple class of multivariate GARCH models. Journal of Business \& Economic Statistics, 20, 339-350.

27. Engle, R. F., \& Kelly, B.T. (2008). Dynamic Equicorrelation. Mimeo, SternSchool of Business.

28. Hosking, J.R.M. (1980). The Multivariate Portmanteau Statistic. Journal of the American Statistical Association, 75(371), 602-608.

29. McLeod, A. I., \& Li, W.K. (1983). Diagnostic checking ARMA time series models using squaredresiduals autocorrelations. Journal of Time Series Analysis, 4(4), 269-273. 